\title{
Modelling The Effects of Social Distancing, Antiviral Therapy, And Booster Shots On Mitigating Omicron Spread
}

\author{
Jongmin Lee \\ Konkuk University \\ Renier Mendoza \\ Konkuk University \\ Victoria May P. Mendoza \\ Konkuk University \\ Youngsuk Ko \\ Konkuk University \\ Jacob Lee \\ Hallym University

\section{Yubin Seo} \\ Hallym University \\ Eunok Jung ( $\sim$ junge@konkuk.ac.kr) \\ Konkuk University
}

\section{Research Article}

Keywords:

Posted Date: February 9th, 2022

DOI: https://doi.org/10.21203/rs.3.rs-1322738/v1

License: (9) (i) This work is licensed under a Creative Commons Attribution 4.0 International License. Read Full License 


\section{Modelling the effects of social distancing, antiviral therapy, and booster shots on mitigating Omicron spread}

Jongmin Lee ${ }^{1}$, Renier Mendoza ${ }^{1,2}$, Victoria May P. Mendoza ${ }^{1,2}$, Youngsuk Ko ${ }^{1}$, Jacob Lee ${ }^{3}$, Yubin $\mathrm{Seo}^{3}$, Eunok Jung ${ }^{1, *}$

1 Department of Mathematics, Konkuk University, Seoul, South Korea 2 Institute of Mathematics, University of the Philippines Diliman, Quezon City, Philippines

3 Division of Infectious Disease, Department of Internal Medicine, Kangnam Sacred Heart Hospital, Hallym University College of Medicine

* Correspondence: junge@konkuk.ac.kr

\section{Abstract}

We propose a COVID-19 mathematical model that considers Omicron and previous variants, booster shots, waning, breakthrough infections, and antiviral therapy. We quantify the effects of social distancing (SD) in the Republic of Korea by estimating the reduction in transmission $\mu$ induced by government policies from February 26, 2021 to January 16, 2022. The time-dependent $\mu$ has a value between 0 and 1 , with 1 being the strictest SD. Simulations show that by February 28, 2022, 92\% of infections are caused by Omicron. Strict SD $(\mu=0.81)$ is necessary to reduce the number of cases. However, if the focus is shifted towards reducing the severe instead of daily cases, relaxed SD $(\mu=0.66)$ is possible if the administered booster shots have at least $90 \%$ effectiveness. Furthermore, if the available antiviral pill is at least $89 \%$ effective against severe infections with Omicron, then a more relaxed SD $(\mu=0.54)$ can be implemented.

\section{Introduction}

Designated by the World Health Organization as a variant of concern on November 26, 2021, Omicron is now the dominant variant in several countries including South Africa, Denmark, the United States and the United Kingdom [1]. Omicron has been detected in 149 countries as of January 6, 2022 and continues to pose a high risk for COVID-19 worldwide 2]. Initial studies on Omicron suggest that it is more transmissible [3-5] and has a greater ability to evade immunity than Delta [6,7]. Moreover, Omicron appears to cause less severe infections and a higher chance of reinfection compared to previous variants 2 .

Vaccine effectiveness and immunity induced by vaccines or a prior infection decrease depending on SARS-CoV-2 variants and waning of immunity 8 10]. Despite these, vaccination is still an important measure in protecting the population. Boosters have been rolled out in many countries with priority given to the most vulnerable groups $[2,11,12$. Currently available vaccines remain effective against severe disease and death, can slow down transmission, and may minimize the emergence of new variants [11, 13, 14]. Furthermore, non-pharmaceutical interventions (NPIs), such as wearing masks and social distancing (SD), are crucial in interrupting and delaying the transmission of Omicron 2, 11, 15]. 
The Republic of Korea's COVID-19 pandemic response before vaccination started had centered on SD and Testing/Tracing/Treatment. From February 26, 2021, vaccination rolled out with priority to the elderly and healthcare workers, followed by the younger age groups. A four-tier SD scheme was adopted in July 2021 that outlined the number of people in a gathering and operational guidelines of various facilities [16]. During this time, SD was maintained at Level 2 (SD2) but was raised to Level 4 (SD4) on July 12, 2021, when the fourth wave prompted by the Delta variant began 17. By October 2021, about $75 \%$ of the population had been fully vaccinated and a decline in the number of cases was observed. On November 1, 2021, the government implemented an eased SD level as part of its 'gradual recovery to a new normal' (GR) policy.

A sharp rise in daily cases of COVID-19 was seen towards the end of November until early December 2021 due to the eased SD and dominance of the Delta variant, which was detected in $96 \%$ of tested samples on December [18, 2021. Moreover, breakthrough infections (BTI) comprised $58.2 \%$ of the cases according to the data reported on January 17, $2022[19]$. To maintain a high population-level immunity, more than 100,000 doses of booster shots have been administered per day since November 2021. The duration of getting a booster shot from the second dose had also been shortened from six to three months. Cases with the Omicron variant were first reported in Korea on December 1, 2021. On January 15, 2022, the proportion of infections with Omicron was around 26.7\% 19. A stronger SD policy called suspended GR (SGR) had also been reinstated since December 18, 2021. As of January 21, 2022, the average daily confirmed cases and severe patients were 5,101 and 543, respectively. On January 14, 2022, the Pfizer COVID-19 antiviral pill Paxlovid was rolled out in Korea, the first Asian country to do so after authorizing its emergency use in December 20. Clinical trials show that this pill has $89 \%$ effectiveness against severity [21].

Mathematical models have been extensively used to understand the dynamics of COVID-19 in various countries 22 25]. They have also been utilized in proposing strategies to ease the effects of the pandemic 26 28. Furthermore, vaccination roll-out strategies were designed using mathematical models 29 31. Various models considering the Delta variant have been presented 32 34. In this study, we propose a model of the transmission of COVID-19 in Korea considering pre-Delta, Delta, and Omicron variants, waning of immunity, administration of primary vaccine doses and booster shots, and antiviral therapy. We quantify the effects of NPIs induced by the government policies from February 26, 2021 to January 16, 2022 and examine its impact on the daily cases and number of severe patients given the existing vaccination scheme and rising number of Omicron infections. Furthermore, we investigate the effect on the spread of COVID-19 if parameters related to Omicron's transmissibility, severity, and response to vaccines are varied. Through our proposed model, we identify how the SD policy can be eased now that vaccines and antiviral therapy are in place.

\section{Results}

\section{Fitting Results}

The estimation period covered four SD policies: SD2, SD4, GR, and SGR. The GR policy is an eased SD, which is a 'returning to normal' policy. We estimated $\mu(t)$, which quantifies the NPIs according to SD policies of the government. Figure 1 Panels (a) and (b) show the best fit of the model to the data on the daily and cumulative confirmed cases (red circles), respectively. The vertical lines mark the changes in SD policy. The model captured the trend of cases during SD2 and SD4, and the sharp rise in daily cases during the GR phase which peaked on December 19, 2021. A steep decline was seen shortly after SGR was implemented and following the model's trend, a slow 
(a)

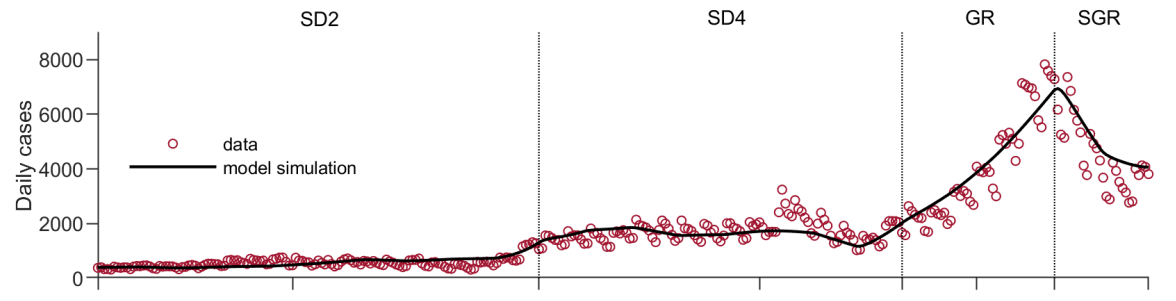

(b)

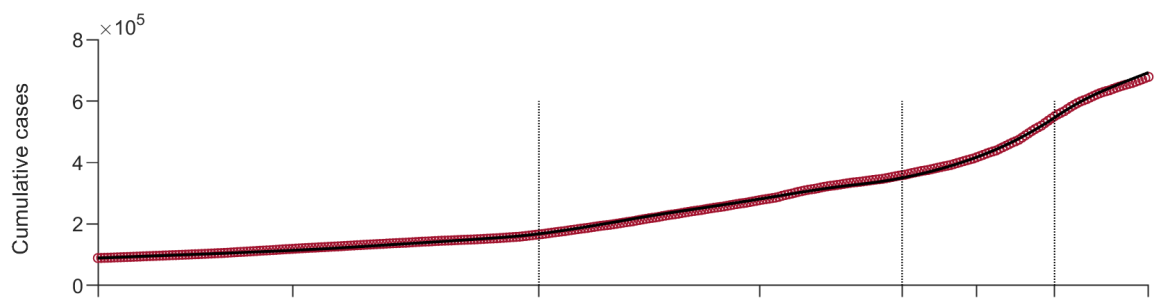

(c)

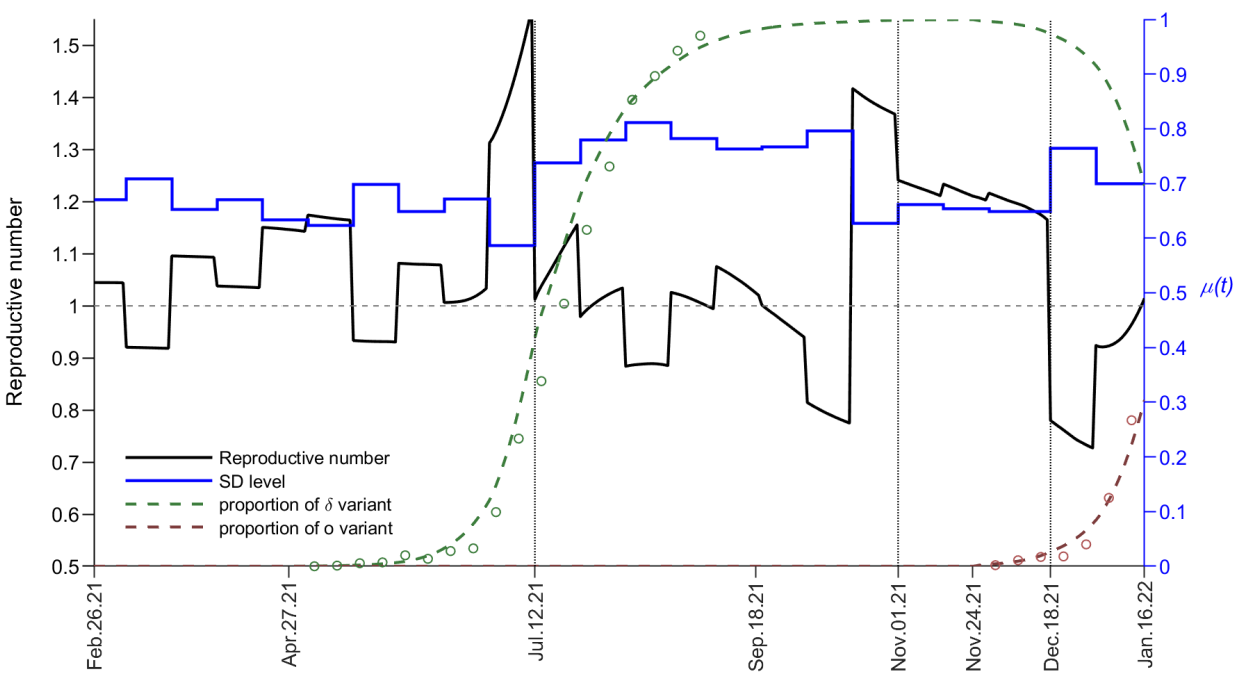

Figure 1. Parameter-fitting results for $\mu(t)$ from February 26, 2021 to January 16, 2022. The vertical dotted lines depict the transition between the different SD policies. Panels (a) and (b) show the best fit of the model to the daily and cumulative confirmed cases, respectively. The red circles represent the data on daily and cumulative cases. Panel (c) shows the effective reproductive number $\mathcal{R}(t)$ (black curve), estimated values of $\mu(t)$ (blue curve), and proportion of Delta and Omicron variants among the confirmed cases (green and brown dashed curves, respectively). The green and brown circles indicate the data on the proportion of Delta and Omicron infections among tested samples, respectively.

decrease in daily cases was observed.

The black and blue curves in Panel (c) show the calculated $\mathcal{R}(t)$ and estimated $\mu(t)$ values, respectively. The green and brown circles and dashed curves in Panel (c) depict the data and model fit on the proportion of infections with the Delta and Omicron variants, respectively. During SD2, $\mathcal{R}(t)$ remained on a range close to 1 except during the two weeks (including Chuseok, the second largest holiday in Korea) before the implementation of SD4, where $\mathcal{R}(t)$ jumped to 1.54. On the same two-week interval, the lowest value of $\mu(t)=0.59$ was estimated and the proportion of cases with the Delta variant (green curve) began to increase steeply. The values of $\mu(t)$ during SD2 varied 
between 0.59 and 0.71. From July 12 to October 31, 2021, Delta became the dominant variant of SARS-CoV-2. The values of $\mu(t)$ ranged from 0.63 to 0.81 . Its value dropped to 0.63 in the last two weeks before the policy was changed to GR, and consequently, $\mathcal{R}(t)$ increased to 1.41. During the eased GR phase, $\mu(t)$ and $\mathcal{R}(t)$ remained at around 0.65 and 1.2, respectively. When the enhanced policy (SGR) was reinstated on December 18, 2021, the proportion of cases with the Omicron variant was only around $4 \%$. The estimated $\mu(t)$ increased to about 0.73 and $\mathcal{R}(t)$ dropped to 0.7 . However, $\mathcal{R}(t)$ is observed to be increasing towards the end of the estimation period. Table 1 presents the average and range of $\mu(t)$ estimates obtained on each SD phase.

\begin{tabular}{cccc}
\hline Dates & SD Level & Average $\mu(t)$ & Range of $\mu(t)$ \\
\hline Feb 26 to Jul 11, 2021 & SD2 (Level 2) & 0.66 & $(0.59,0.71)$ \\
\hline Jul 12 to Oct 31, 2021 & SD4 (Level 4) & 0.76 & $(0.63,0.81)$ \\
\hline Nov 1 to Dec 18, 2021 & GR (Gradual Recovery) & 0.65 & $(0.65,0.66)$ \\
\hline Dec 19, 2021 to Jan 16, 2022 & SGR (Suspended GR) & 0.73 & $(0.70,0.76)$ \\
\hline
\end{tabular}

Table 1. The average and range of the estimated $\mu(t)$ values on each SD phase from February 26, 2021 to January 16, 2022.

\section{Waning and Breakthrough Infections}

Figure 2 shows the changes in the proportion of the uninfected (Panel (a)) and infected (Panel (b)) classes as more people were vaccinated and different SARS-CoV-2 variants arrived in Korea. The vertical line on January 16, 2022 marks the start of the forecasting period. At the beginning of the simulation, almost all of the uninfected classes were unprotected. From July 12 to October 31, 2021, the proportion of vaccinated individuals increased considerably from around $20 \%$ to $70 \%$. This is consistent with the data on vaccine coverage (11.8\% to $75 \%$ 35 36]). Consequently, the proportion of fully-protected individuals (dark blue) in the same period increased, and the waning class (light blue) constituted about one-fifth of the uninfected population in the subsequent GR phase. Since the administration of booster shots started in October 2021, the proportion of fully-protected individuals increased steeply. Extending the period until February 28, 2022, simulations show that the number of protected individuals (blue regions) will reach about $60 \%$ of the vaccinated population, assuming that the number of daily administered booster shots is 450,000. As more people receive booster shots, the proportion of individuals with waned immunity is reduced from $25 \%$ on December 18, 2021 to $10 \%$ on February 28, 2022.

Figure 2 Panel (b) shows the proportion of BTI (red regions) and non-BTI (green regions) by different SARS-CoV-2 variants. The black dots represent the proportion of BTI from the data [19 and these are captured by the model results on BTI with Delta. The proportion of BTI with Delta increased until the end of GR and decreased thereafter, as the proportion of BTI with Omicron began to rise. Assuming that SGR is continued until the end of forecast period, approximately $92 \%$ of infections (BTI and non-BTI) are Omicron, and almost one-fourth of individuals infected with Omicron are unvaccinated.

\section{Model Forecasts}

Figure 3 Panel (a) shows the model forecasts for the number of daily and severe cases when $\mu$ is varied. The green curve in Panel (a) corresponds to $\mu_{\max }=0.81$, while the 
(a)

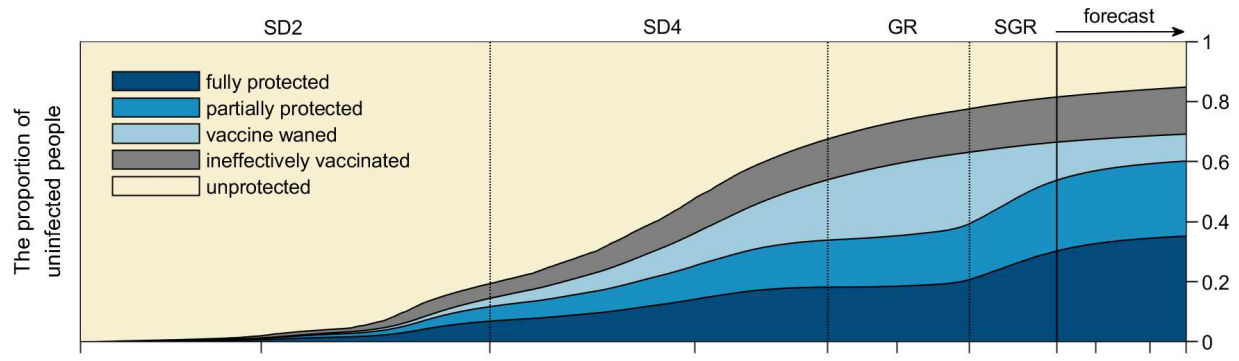

(b)

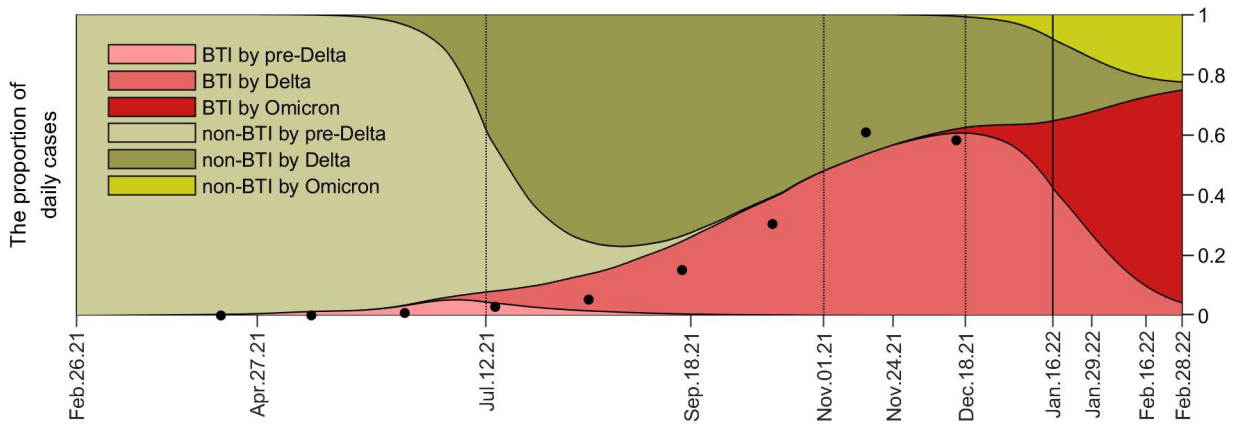

Figure 2. The proportion of the uninfected population and breakthrough infections (BTI) from February 26, 2021 to February 28, 2022. Panel (a) shows how the proportions of different uninfected classes change over time. Panel (b) illustrates the proportions of non-BTI and BTI caused by the different variants. The total proportion of BTI from the available data is also shown (black dots). The black dotted lines depict the transition between the different SD policies. The solid black line on January 16, 2022 indicates the end of the estimation and start of the forecast period.

magenta and black curves correspond to $\mu=0.65$ (GR) and $\mu=0.73$ (SGR). The gray curves correspond to the cases when $\mu$ is varied from 0.65 to $\mu_{\max }$. In this illustration, we assume that the number of daily booster shots is 450,000 . In both GR and SGR scenarios, daily cases will exceed the maximum recorded number of 7,828 cases by February 28, 2022. The threshold on the number of severe beds in Korea equal to 2,145 is reached at the GR level. Moreover, daily and severe cases may balloon to 50,000 and 3,000 , respectively. If $\mu>0.79$, which translates to an SD level more strict than SGR, then a decreasing trend in daily cases until February 28, 2022 is observed. In comparison, if $\mu>0.77$, then the trend for severe cases is decreasing.

Figure 3 Panel (b) shows the model forecast for the number of daily and severe cases when the number of booster shots administered from January 17, 2022 is 0, 450,000 , or 800,000 daily. If $\mu=0.73$ (SGR), then the number of daily cases will rise even if 800,000 booster shots are administered daily (blue curve). If SD is enhanced to $\mu_{\max }$, the number of cases will go down. In both $\mu$ values, increasing the amount of daily administered booster shots decreases the overall number of infections. At SGR, daily cases may rise to 12,700 when no booster shots are given compared to 9,600 when 450,000 booster shots are administered daily.

Figure 4 shows the forecast for the proportion of vaccinated (red bars) and unvaccinated (green bars) individuals, with respect to the total vaccinated and unvaccinated populations, respectively, who will be infected on February 28, 2022 under different SD scenarios: $\mu=0.59,0.65,0.73$, and 0.81 . In this simulation, the number of daily administered booster shots from January 17, 2022 is set to 450,000. In all four values of $\mu$, the proportion of new cases of unvaccinated individuals is more than double 
(a) $\mu$ scenarios
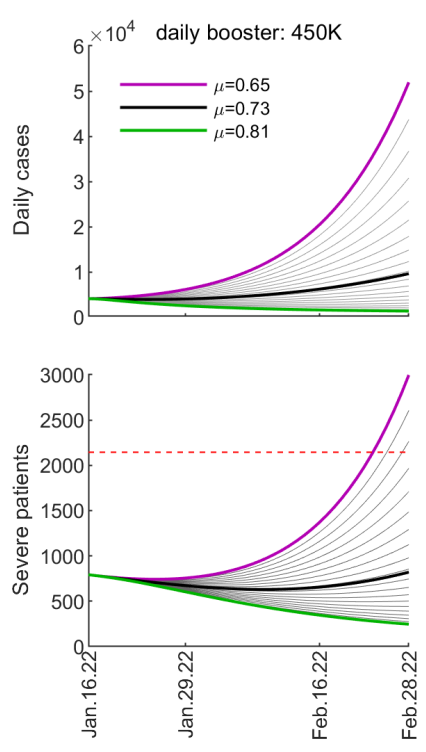

(b) daily booster shot scenarios

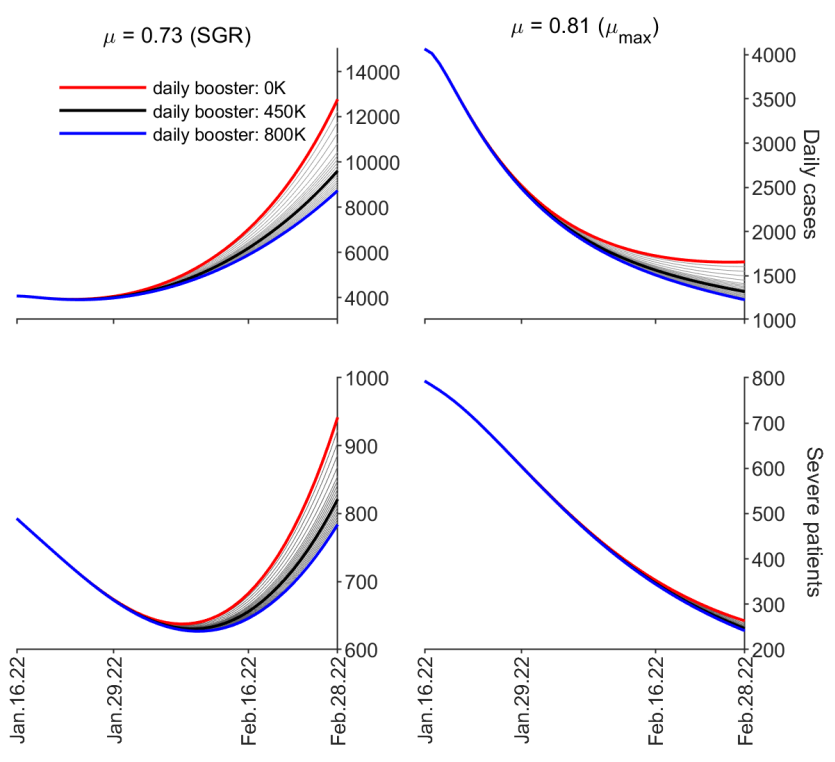

Figure 3. Forecast for the number of daily and severe cases considering different (a) values of $\mu(t)$ corresponding to different SD levels and (b) amount of daily administered booster shots when $\mu=0.73$ (SGR) and $\mu=0.81$ (maximum estimated $\mu$ that occurred in SD4).
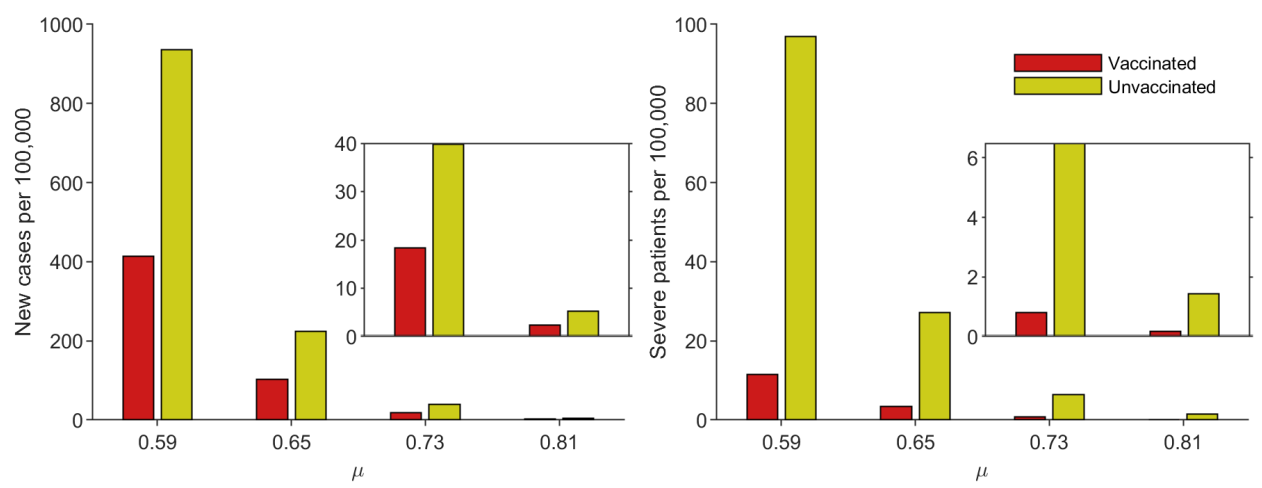

Figure 4. Predicted proportion of vaccinated and unvaccinated individuals who will be infected by the end of the forecast period on February 28, 2022 under different $\mu$ values. The plot on the left shows the predicted number of new cases per 100,000 people of unvaccinated (green bars) and vaccinated (red bars) individuals when $\mu=$ $0.59,0.65,0.73$, and 0.81 . The plot on the right is the predicted number of severe patients per 100,000 people under different $\mu$ values. The insets show more closely the bar graphs when $\mu=0.73$ and $\mu=0.81$.

than that of the vaccinated and the difference in proportions is eight-fold for the severe patients. In particular, when $\mu=0.73$ (SGR), the predicted BTI is 18 per 100,000 vaccinated individuals and 0.8 of these cases are severe. Moreover, 40 infections are predicted per 100,000 unvaccinated individuals and 6.5 of these are severe cases.

In Figure 5 we investigate the effect of different $\mu$ values to the maximum number 

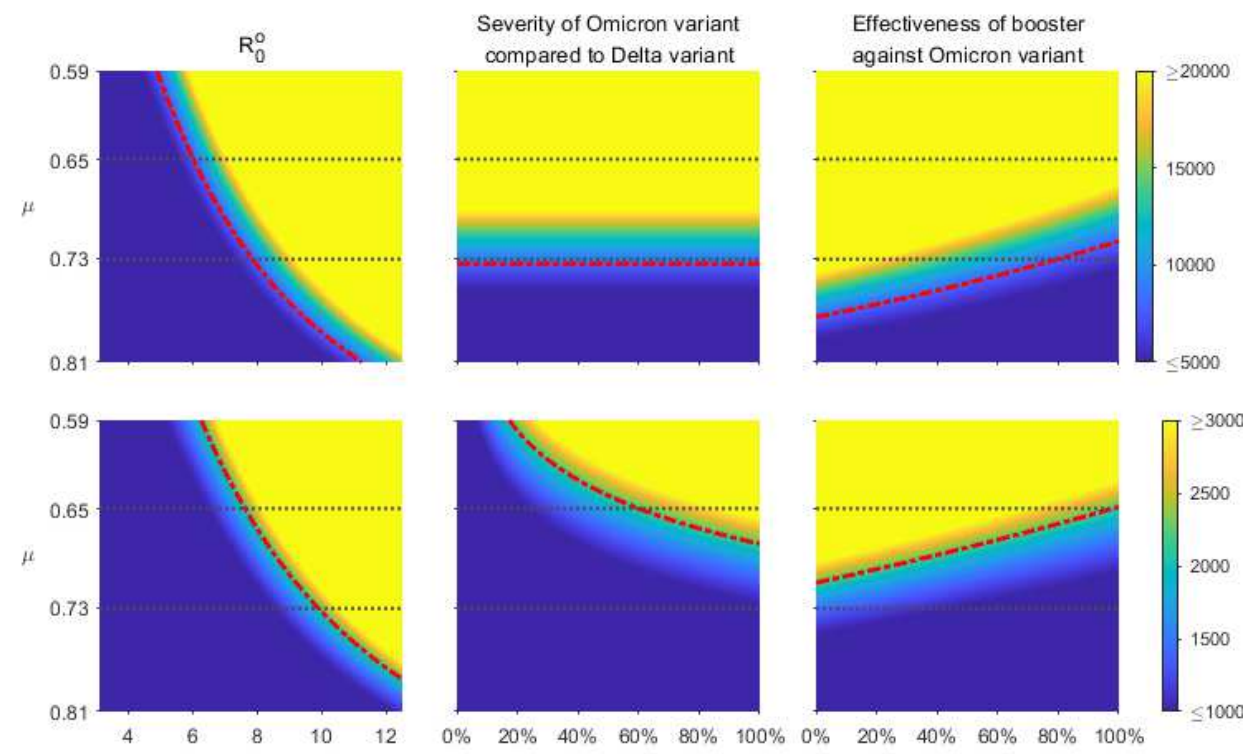

Figure 5. Predicted maximum number of daily cases and severe patients considering transmissibility and severity of Omicron, and effectiveness of the booster shots during the period of January 17, 2022 to February 28, 2022. Simulations are done using varying $\mu$, which quantifies different SD levels. The red dashed lines indicate a threshold for the maximum number of daily cases and severe patients to be more than 7,828 and 2,145, respectively. The black dashed lines at $\mu=0.65$, and $\mu=0.73$ correspond to GR and SGR levels, respectively.

of daily and severe cases under various assumptions on Omicron's transmissibility, severity relative to the Delta variant, and response to booster shots. Transmissibility is quantified using the basic reproductive number. In the first column, we vary $\mathcal{R}_{0}^{o}$ and fix the proportion of cases that becomes severe and effectiveness of booster shots to their values shown in Table 2. Similarly, different values of disease severity and effectiveness of booster shots were considered in the second and third columns, respectively. If Omicron is twice as transmissible as Delta $\left(\mathcal{R}_{0}^{o}=2 \mathcal{R}_{0}^{\delta}\right)$, a strict SD policy $(\mu>0.79)$ ensures that the number of severe patients is below the threshold. If $\mathcal{R}_{0}^{o}$ is 1.3 times as transmissible as Delta 37], GR $(\mu=0.65)$ may be the appropriate SD policy.

Regardless of the effectiveness of booster shots, SD level should be at least SGR $(\mu=0.73)$ to maintain the severe cases at manageable numbers. Meanwhile, SD level can be relaxed to GR as long as booster shots are highly effective. This can be achieved if the booster shots are Omicron-specific [38. In particular, if the effectiveness of booster shots is $90 \%$, then at $\mu=0.66$ the number of severe cases will not exceed the threshold. If the proportion of severe infections with Omicron is less than $20 \%$ of the proportion with Delta, that is, $p_{3}<0.2 p_{2}$, then $\mu$ can be set to 0.6 , which corresponds to a level that is more relaxed than GR.

Antiviral therapy reduces the number of severe cases. Figure 6 shows the predicted number of severe patients by February 28, 2022 under different $\mu$ values given that antiviral therapy is given to all infected and effectiveness of the pill against severity is $89 \% 39,40$. Compared to the projections without the antiviral therapy (see Figure 3 Panel (a), bottom image), the number of severe cases in this simulation will not reach the threshold even if SD level is GR. The black curve on Panel (b) of Figure 6 shows the 

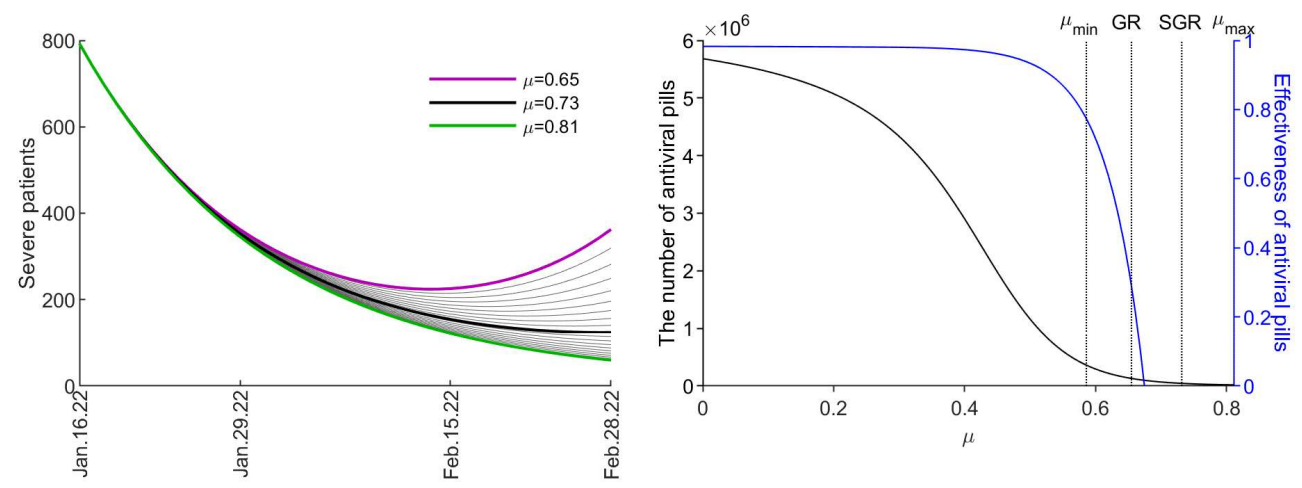

Figure 6. Incorporating antiviral pill in the forecasts. Panel (a) shows the number of severe patients considering different values of $\mu(t)$ corresponding to different SD levels if infected individuals are given antiviral pills. Panel (b) illustrates the minimum number and effectiveness of the antiviral pill so that the number of severe cases will not reach the threshold at the end of the simulation. For this simulation, we also consider the extreme case of $\mu=0$ (without intervention) and $\mu=0.81\left(\mu_{\max }\right)$.

required minimum number of pills under each $\mu$ value to maintain severe cases below threshold. As expected, as SD policy is eased, more pills will be necessary. The blue curve in Panel (b) of Figure 6 shows how the SD level can be chosen depending on the effectiveness of the pill. If the antiviral pill is $89 \%$ effective against hospitalization, then $\mu=0.54<\mu_{\min }$, which means that the policy can be eased to a level that is more relaxed than SD2. If the antiviral pill is ineffective against Omicron, $\mu$ should be at least 0.67 , which corresponds to an SD level stricter than GR.

\section{Discussion}

Until December 2021, the characteristics of the Omicron variant, such as transmissibility and severity, remain uncertain [37,41,42. Mathematical modelling is an effective tool in investigating possible scenarios in these uncertain situations. Through our modelling study, we analyze the effects of booster shots, antiviral therapy, and NPIs that the Korean government may consider to control the spread of new variants.

The rise in the daily confirmed cases observed in July was influenced by the spread of the Delta variant, which we assumed has twice the basic reproductive number of pre-Delta variants. The Korean government raised the SD level to SD4 before the proportion of infections with the Delta variant reached $40 \%$. Despite of the SD4, the reproductive number during early SD4 increased to more than one since the proportion of the Delta variant soared to more than $80 \%$. When Delta became the dominant variant in Korea and $\mu(t)$ value was kept high, the reproductive number fell below one. However, the reproductive number increased to about 1.1 in mid-September, around the Korean Thanksgiving Day celebration. At the end of SD4, the reproductive number jumped to 1.4 because of Halloween and anticipation of the scheduled easing of SD policy on November 1.

Although the average $\mu(t)$ values in SD2 and GR phases are similar (see Table 1), the average reproductive number in GR is larger than in SD2 since Delta was dominant during GR but not during SD2. Consequently, daily confirmed cases increased from about 2,000 to 7,000 within 40 days during GR compared to about 400 to 1000 cases within 130 days during SD2. Similarly, the average value of $\mu(t)$ during SGR is similar 
to SD4, yet the number of infections seems to be decreasing during SGR, unlike in the fourth wave that occurred in July. Possible reasons for this difference are that the SD was enhanced before the proportion of Omicron cases reached 1\%. Also, more than 95\% of the adult population had already been vaccinated by December 2021, and more than 400,000 booster shots are continued to be administered daily. Hence, the cases did not increase even though the basic reproductive number of Omicron is assumed to be 1.3 times higher than that of Delta and the effectiveness of vaccines against Omicron is reduced by more than $30 \%$. However, if the SD policy is eased and the proportion of Omicron increases, cases may rise again.

From July 12 to December 18, 2021, Delta became the dominant variant and the proportion of BTI increased to more than $60 \%$. By December 18, 2021, one-fourth of uninfected individuals have waned immunity. As more people were given booster shots from December 18, 2021 to January 16, 2022, the proportion of fully protected individuals increased by $10 \%$ while the proportion of waning group is reduced by $13 \%$. As a result, the proportion of BTI only increased by $2 \%$. From January 16 to February 28, 2022, Omicron may become the dominant variant. Because the effectiveness of booster shots against Omicron is lower than Delta, the proportion of BTI is projected to increase.

If $\mu(t)=0.65$ (GR) and at least $64 \%$ of the population is fully protected, then $\mathcal{R}(t)$ will be less than one (refer to Equation 1). However, by the end of our forecast period, the proportion of people with full protection is less than $40 \%$. Moreover, one-fourth of infections are unvaccinated individuals. Hence, booster shots to those whose immunity waned and primary shots to those who remained unvaccinated should be encouraged to reduce Omicron infections. Since the vaccination coverage of adults in Korea is at $96 \%$, then the majority of unvaccinated individuals are underage 43]. The government may consider vaccinating the underage group once safe and effective vaccines are available.

Results in Figure 4 suggest that vaccination reduces the probability of severe infections and as SD is enhanced, BTI and non-BTI can be minimized. Although both $\mu=0.73$ (SGR) and $\mu=0.81$ scenarios with 450,000 daily administered booster shots indicate that the number of daily cases and severe patients are still below threshold values by February 28, 2022 (see Figure 3), the model's trend is still increasing when $\mu=0.73$ but decreasing when $\mu=0.81$. This suggests while keeping a high level of population immunity is important, maintaining enhanced NPIs is also necessary to suppress the number of Delta and Omicron infections. Simulations in Figure 5 suggest that if the severity of Omicron is reduced and booster shots with high effectiveness against Omicron infection are used, then SD policy can be relaxed. Hence, the availability of Omicron-specific booster shots 38 to the population would be crucial in easing restrictions.

Since Pfizer COVID-19 antiviral pills are already being deployed in Korea [20, the proportion of severe cases may be further reduced. Our simulations show that if antiviral pill is provided to COVID-19 patients, severe cases is reduced and SD policy can be eased. However, a more relaxed policy translates to more cases and hence, procurement of more pills. Considering a limited supply amidst global competition, our model can be used as a guide to determine the appropriate level of SD given the constraint on the availability of pills. Should the government decide to implement GR policy, the number of cases will soar. However, with the vaccine and antiviral therapy interventions, the number of severe cases can be manageable.

Our study does not take into account age-related differences in disease severity and prioritization in vaccination. An age-structured model would entail age-specific Omicron data which are insufficient as of writing. Since there is still uncertainty on the basic reproductive number and severity of Omicron, and vaccine effectiveness against Omicron, ranges of values related to these parameters based on initial studies were 
considered when the scenarios on different $\mu(t)$ and number of daily administered booster shots were simulated. In our forecasts, we assume that the SD policy on January 16, 2022 is continued and the value of $\mu(t)$ is the same from January 17 to February 28, 2022. Changes in people's contact patterns during holidays such as the Lunar New Year are not considered. Our model assumes that all infected individuals receive the pill and does not consider prioritization on antiviral therapy. A further study on antiviral pill allocation is an interesting research direction. Although our proposed model is only applied to Korea, it is general enough that it can also be used to other countries.

In summary, we have presented a model that describes the dynamics of the infected and uninfected population as vaccination rolled out, variants emerged, and SD policies changed. Our forecasts suggest that in the next two months, most of the cases in Korea will be caused by Omicron. The model shows that as the vaccine-induced immunity wanes, the proportion of BTI increases. Moreover, the results indicate that booster shots offer added protection against infection and reinfection, and highly effective vaccines can reduce the number of severe cases. The unvaccinated population is more vulnerable to infection, given that they do not have added protection against severity. In addition to NPIs, administration of vaccines and booster shots to as many people as possible remains a key strategy in easing the effects of the pandemic. Furthermore, antiviral therapy, if effective, offers further reduction on the number of severe patients. With the vaccination program and antiviral therapy in place, our study shows that a return to a more relaxed SD policy is possible.

\section{Materials and Methods}

\section{Data}

We use the Republic of Korea's data on vaccination containing the number of primary doses and booster shots from February 26, 2021 to January 16, 2022 35, 36, 44]. The proportion of administered vaccine type are applied to the calculation of model effectiveness 45]. We aggregated the daily number of confirmed cases, seriously-ill patients, and deaths, from the Korea Disease Control and Prevention Agency (KDCA) reports, excluding confirmed cases in Lazaretto [19. Data on the proportion of BTI among confirmed cases with pre-Delta, Delta, and Omicron variants were obtained from KDCA [18, 19].

\section{Mathematical Model}

We use an SEIR model to describe the transmission of COVID-19 considering the variants, vaccination, waning, and booster shots. The model diagram in Figure 7 shows that once susceptible individuals $S$ are vaccinated, then they are categorized into effectively $(V)$ or ineffectively $(U)$ vaccinated groups. The parameter $\theta$ denotes the number of primary doses of vaccines administered daily, according to available data. We assume that individuals in $U$ cannot get immunity from vaccines. After an average of $1 / \omega$ days, individuals in $V$ become protected $\left(P_{j}\right)$ against pre-Delta, Delta, or Omicron variants. Here, $j=1,2$ or 3 denote infection with pre-Delta, Delta, and Omicron, respectively. We assume that individuals who are fully protected from Omicron are also protected from the other variants, and individuals who are fully protected from Delta are also protected from the pre-Delta variants. Individuals in $P_{1}$ may be infected by Delta or Omicron variants. As the immunity provided by vaccines wanes over time, the protected individuals move to the $W$ compartment at a waning rate $\tau_{j}^{v}$. When individuals in $W$ receive booster shots, they move to the $V_{b}$ compartment and become protected again after an average of $1 / \omega_{v}$ days. Similarly, $\theta_{v}$ represents the number of 


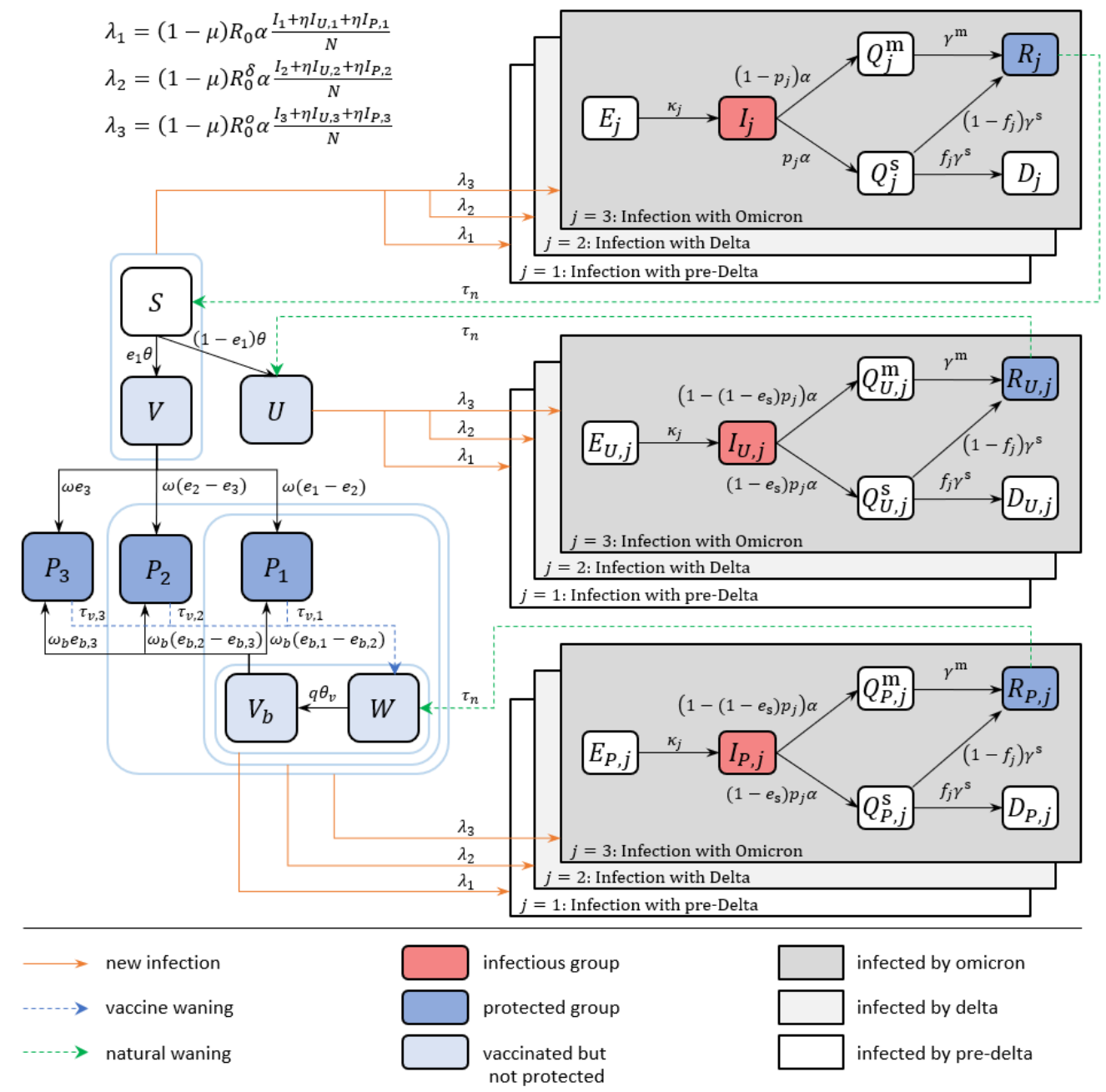

Figure 7. The model flowchart describing the transmission of the COVID-19 considering different variants, vaccination, waning, and booster shots.

daily administered booster shots according to the data. We assume that only $q \%$ of $\theta_{v}$ are actually administered to the $W$ group and the rest are given to individuals in $U$. The parameters $e_{i}$ and $e_{b, j}$ denote the adjusted effectiveness of primary and booster doses, respectively. Vaccine-induced immunity is assumed to wane exponentially. The calculated values of $e_{i}, e_{b, j}, \omega, \tau_{v, j}$, and $f$ are shown in Table 2 and the details of the calculation are given in Supplementary A.

Individuals in the susceptible and vaccinated compartments may be exposed $\left(E_{j}, E_{U, j}, E_{P, j}\right)$ to any of the variants with forces of infection $\lambda_{j}(t)$. The expression $1-\mu(t)$ in $\lambda_{j}(t)$ accounts for the reduction in transmission induced by NPIs such as social distancing. The basic reproduction numbers of pre-Delta, Delta, and Omicron are denoted as $\mathcal{R}_{0}, \mathcal{R}_{0}^{\delta}$, and $\mathcal{R}_{0}^{o}$, respectively. Exposed individuals become infectious $\left(I_{j}, I_{U, j}, I_{P, j}\right)$ after $1 / \kappa_{j}$ days on average. For all variants, we assume that vaccinated infectious hosts have reduced transmissibility, with a reduction rate $\eta$, compared to unvaccinated infectors. We assume that the value of $\eta$ is $23 \%$ for Delta and $4.3 \%$ for Omicron [46]. Once confirmed, infectious individuals are isolated and classified as mild $\left(Q_{j}^{\mathrm{m}}, Q_{U, j}^{\mathrm{m}}, Q_{P, j}^{\mathrm{m}}\right)$ or severe $\left(Q_{j}^{\mathrm{s}}, Q_{U, j}^{\mathrm{s}}, Q_{P, j}^{\mathrm{s}}\right)$. A quarantined individual either recovers 
$\left(R_{j}, R_{U, j}, R_{P, j}\right)$ after $1 / \gamma^{\mathrm{m}}$ or $1 / \gamma^{\mathrm{s}}$ days on average, or dies $\left(D_{j}, D_{U, j}, D_{P, j}\right)$ from the disease with fatality rate $f$. Because vaccination protects against getting severe, the proportion of severe cases $p_{j}$ is reduced by a factor $e^{\mathrm{s}}$. Individuals who recover from the disease develop natural immunity against COVID-19 until their immunity wanes. In this study, we set the natural recovery waning period $1 / \tau_{n}$ as 4 months 47.50 . The model parameters are listed in Table 2 and the model equations are in Supplementary B.

\begin{tabular}{|c|c|c|c|c|c|}
\hline Symbol & Description (units) & pre- $\delta$ & $\delta$ & $o$ & Ref. \\
\hline $\mathcal{R}_{0}, \mathcal{R}_{0}^{\delta}, \mathcal{R}_{0}^{o}$ & Basic reproduction number & 3.17 & 6.24 & 8.12 & \begin{tabular}{|l|l|}
37,51 & \\
52 & \\
\end{tabular} \\
\hline $1 / \kappa_{j}$ & Mean latent period (days) & 4 & 2 & 2 & \begin{tabular}{|l|l|}
53 & 54 \\
\end{tabular} \\
\hline $1 / \alpha$ & Mean infectious period (days) & 6 & 6 & 6 & \begin{tabular}{|l|l|}
54 & 55 \\
\end{tabular} \\
\hline$p_{j}$ & Proportion of severe cases & $2.28 \%$ & $2.28 \%$ & $1.82 \%^{*}$ & \begin{tabular}{|l|l|}
42 & 56 \\
\end{tabular} \\
\hline$f$ & $\begin{array}{c}\text { Mean fatality rate among severe } \\
\text { cases }\end{array}$ & $43.9 \%^{\dagger}$ & $43.9 \%^{\dagger}$ & $43.9 \%^{\dagger}$ & \begin{tabular}{|l|l|}
44 & 56 \\
\end{tabular} \\
\hline $1 / \gamma^{m}$ & $\begin{array}{l}\text { Mean duration of hospitalization } \\
\text { for mild cases (days) }\end{array}$ & 11.7 & 11.7 & 11.7 & \begin{tabular}{|l|l|}
57 & 58 \\
\end{tabular} \\
\hline $1 / \gamma^{s}$ & $\begin{array}{l}\text { Mean duration of hospitalization } \\
\text { for severe cases (days) }\end{array}$ & $14^{*}$ & $14^{*}$ & $14^{*}$ & 5960 \\
\hline$\tau_{n}$ & $\begin{array}{c}\text { Mean waning rate of } \\
\text { infection-induced immunity } \\
(1 / \text { day })\end{array}$ & $1 / 120$ & $1 / 120$ & $1 / 120$ & \begin{tabular}{|l|l|}
47 & 50 \\
\end{tabular} \\
\hline$\tau_{v, j}$ & $\begin{array}{c}\text { Mean waning rate of } \\
\text { vaccine-induced immunity } \\
(1 / \text { day })\end{array}$ & $1 / 297^{\dagger}$ & $1 / 297^{\dagger}$ & $1 / 109^{\dagger}$ & \begin{tabular}{|l|l|}
45 & 61 \\
\end{tabular} \\
\hline$e_{j}$ & $\begin{array}{l}\text { Adjusted effectiveness of mRNA } \\
\text { and viral vector vaccines }\end{array}$ & $84.8 \%^{\dagger}$ & $84.8 \%^{\dagger}$ & $53.2 \%^{\dagger}$ & \begin{tabular}{|l|l|}
45 & 61 \\
\end{tabular} \\
\hline$e_{b, j}$ & $\begin{array}{l}\text { Adjusted effectiveness of booster } \\
\text { vaccines }\end{array}$ & $96.0 \%^{\dagger}$ & $96.0 \%{ }^{\dagger}$ & $66.8 \% \%^{\dagger}$ & \begin{tabular}{|l|l|}
45 & 61 \\
\end{tabular} \\
\hline$e^{s}$ & $\begin{array}{l}\text { Vaccine effectiveness against } \\
\text { severe infections }\end{array}$ & $73 \%$ & $73 \%$ & $73 \%$ & 56 \\
\hline$\eta$ & $\begin{array}{l}\text { Vaccine-induced reduction in } \\
\text { transmission }\end{array}$ & $23 \%$ & $23 \%$ & $4.2 \%$ & 46 \\
\hline $1 / \omega$ & $\begin{array}{l}\text { Mean duration to have immune } \\
\text { after normal vaccines (days) }\end{array}$ & $40^{\dagger}$ & $40^{\dagger}$ & $40^{\dagger}$ & \begin{tabular}{|l|l|}
45 & 61 \\
\end{tabular} \\
\hline $1 / \omega_{b}$ & $\begin{array}{l}\text { Mean duration to have immune } \\
\text { after booster vaccines (days) }\end{array}$ & 21 & 21 & 21 & 61 \\
\hline $1 / e_{\text {pill }}$ & $\begin{array}{l}\text { Effectiveness of antiviral pills } \\
\text { against severity }\end{array}$ & $89 \%$ & $89 \%$ & $89 \%$ & 39 \\
\hline
\end{tabular}

Table 2. All symbols and values used in the mathematical model. The subscript $j=1,2,3$ refers to infection with pre-Delta, Delta, and Omicron variants, respectively. ${ }^{*}$ : assumed value, $\dagger$ : calculated value) 


\section{Parameter Estimation}

The time-dependent parameter $\mu(t)$ is fitted by minimizing the square error of the cumulative number of cases using the model $\int \sum_{j=1}^{3} \alpha\left(I_{j}+I_{U, j}+I_{P, j}\right)$ and aggregated data from February 26, 2021 to January 16, 2022 from the KDCA presses 35, 36, 44. We assume that the value of $\mu(t)$ changes every two weeks. The least-squares formulation is solved using the MATLAB built-in function lsqcurvefit. The initial values of individuals exposed to Delta and Omicron variants when the first cases of each variant are confirmed are set to 1 and 100, respectively. These are estimated by fitting the model to the available data on the proportion of variants among confirmed cases 18, 19. We set the date of first confirmed case of the Delta and Omicron variants to April 27, 2021 and November 24, 2021, respectively 62 64.

The effective reproductive number $\mathcal{R}(t)$ is calculated using next generation method 65, 66]. We obtain

$$
\begin{aligned}
\mathcal{R}(t)= & \mathcal{R}_{0}(1-\mu(t)) \frac{S+V+U+W+V_{b}}{N} \frac{I_{1}+\eta\left(I_{U, 1}+I_{P, 1}\right)}{\sum_{j=1}^{3}\left(I_{j}+I_{U, j}+I_{P, j}\right)}+ \\
& \mathcal{R}_{0}^{\delta}(1-\mu(t)) \frac{S+V+U+W+V_{b}+P}{N} \frac{I_{2}+\eta\left(I_{U, 2}+I_{P, 2}\right)}{\sum_{j=1}^{3}\left(I_{j}+I_{U, j}+I_{P, j}\right)}+ \\
& \mathcal{R}_{0}^{o}(1-\mu(t)) \frac{S+V+U+W+V_{b}+P+P_{\delta}}{N} \frac{I_{3}+\eta\left(I_{U, 3}+I_{P, 3}\right)}{\sum_{j=1}^{3}\left(I_{j}+I_{U, j}+I_{P, j}\right)} .
\end{aligned}
$$

\section{Modelling Scenarios}

Based on the model, the uninfected population can be divided into five groups: fully protected against all the variants $\left(P_{3}\right)$, partially protected against the variants $\left(P_{1}, P_{2}\right)$, vaccine waned population $\left(W, V_{b}\right)$, ineffectively vaccinated $(U)$, and unprotected $(S, V)$. The infected groups are divided according to variant and whether it is BTI or non-BTI. We look at the dynamics of the proportions of the uninfected and infected groups as more individuals were vaccinated with primary and booster doses, and as the variants emerged.

From the data-fitted values of the time-dependent parameter $\mu(t)$, we denote as $\mu_{\max }$ and $\mu_{\min }$ the highest and lowest values of $\mu(t)$ and associate these values to strict and eased SD policies, respectively. We perform simulations from January 17 to February 28, 2022, to predict the number of daily cases and severe patients by varying $\mu$, representing different SD levels. We also examine the effect of booster shots on the daily cases and number of severe patients by varying the number of daily booster shots administered from January 17, 2022 and assuming different levels of SD. We set the threshold for the number of daily cases and severe beds to 7,828 and 2,145, respectively, which correspond to the maximum recorded daily cases and available number of severe beds in Korea. Furthermore, we calculate the proportion of infections from the vaccinated and unvaccinated populations considering different SD levels by the end of the forecast period.

Simulations are also performed to examine the impact of Omicron's transmissibility, disease severity relative to Delta, and effectiveness of booster shots against Omicron to the maximum number of daily cases and severe patients under different assumptions on the level of SD. Until December 2021, there is limited information on the basic reproductive number of Omicron. Early studies suggest that Omicron is more 
transmissible than Delta $\left.\left(\mathcal{R}_{0}^{o}>\mathcal{R}_{0}^{\delta}\right)\right] 3$ and that $R_{0}^{o}$ could be 1.3 times $\mathcal{R}_{0}^{\delta} 37$. In the simulations, we consider values of $\mathcal{R}_{0}^{o} \in\left[0.5 \mathcal{R}_{0}^{\delta}, 2 \mathcal{R}_{0}^{\delta}\right]$. Since the severity of COVID-19 caused by Omicron may be different compared to Delta [67] or can be reduced by an effective antiviral pill, we consider values of $p_{3}$ from $0 \%$ to $100 \%$. Recent studies have estimated the effectiveness of booster shots in preventing Omicron infections $7,68,69$ but these values may change as more data are studied. Although preliminary studies suggest that booster shots may not be as effective against Omicron compared to Delta, Omicron-specific booster shots [38] maybe soon available which can increase the effectiveness of vaccines. Hence, booster shot effectiveness against Omicron is varied from $0 \%$ to $100 \%$.

Since the Korean government started to administer COVID-19 antiviral pills [20], we also predict the number of severe cases by February 28, 2022 if infected individuals are given antiviral therapy. We assume that the effectiveness of the drug against severity is $89 \%$ 39. We incorporate this into the model by multiplying the expression $(1-0.89)$ to the proportion of severe cases $p_{j}$. Under different assumptions on drug effectiveness, the minimum number of antiviral pills necessary to keep the number of severe patients below the threshold is calculated as 10 times the expected number of severe patients without antiviral pills.

\section{References}

1. The New York Times. Tracking Omicron and Other Coronavirus Variants. Accessed 27 January 2022.

2. Switzerland World Health Organization HQ: Headquarters, Geneva. Enhancing response to Omicron SARS-CoV-2 variant: Technical brief and priority actions for Member States (updated from previous version, published 23 December 2021). Technical report, World Health Organization, January 2022. Accessed 27 January 2022 .

3. Ingrid Torjesen. Covid-19: Omicron may be more transmissible than other variants and partly resistant to existing vaccines, scientists fear. BMJ, 2021.

4. Salim S Abdool Karim and Quarraisha Abdool Karim. Omicron SARS-CoV-2 variant: a new chapter in the COVID-19 pandemic. The Lancet, 2021.

5. Shailendra K Saxena, Swatantra Kumar, Saniya Ansari, Janusz T Paweska, Vimal K Maurya, Anil K Tripathi, and Ahmed S Abdel-Moneim. Characterization of the novel SARS-CoV-2 Omicron (B. 1.1. 529) Variant of Concern and its global perspective. Journal of Medical Virology.

6. Juliet R.C. Pulliam, Cari van Schalkwyk, Nevashan Govender, Anne von Gottberg, Cheryl Cohen, Michelle J. Groome, Jonathan Dushoff, Koleka Mlisana, and Harry Moultrie. Increased risk of SARS-CoV-2 reinfection associated with emergence of the Omicron variant in South Africa. medarxiv, 2021.

7. Nick Andrews, Julia Stowe, Freja Kirsebom, Samuel Toffa, Tim Rickeard, Eileen Gallagher, Charlotte Gower, Meaghan Kall, Natalie Groves, Anne-Marie O'Connell, et al. Effectiveness of COVID-19 vaccines against the Omicron (B. 1.1. 529) variant of concern. medRxiv, 2021.

8. Pyoeng Gyun Choe, Chang Kyung Kang, Hyeon Jeong Suh, Jongtak Jung, Kyoung-Ho Song, Ji Hwan Bang, Eu Suk Kim, Hong Bin Kim, Sang Won Park, Nam Joong Kim, et al. Waning antibody responses in asymptomatic and 
symptomatic SARS-CoV-2 infection. Emerging infectious diseases, 27(1):327, 2021.

9. Marc Lipsitch, Florian Krammer, Gili Regev-Yochay, Yaniv Lustig, and Ran D Balicer. SARS-CoV-2 breakthrough infections in vaccinated individuals:

measurement, causes and impact. Nature Reviews Immunology, pages 1-9, 2021.

10. Yair Goldberg, Micha Mandel, Yinon M Bar-On, Omri Bodenheimer, Laurence Freedman, Eric J Haas, Ron Milo, Sharon Alroy-Preis, Nachman Ash, and Amit Huppert. Waning immunity after the BNT162b2 vaccine in Israel. New England Journal of Medicine, 2021.

11. Centers for Disease Control, National Center for Immunization Prevention, and Division of Viral Diseases Respiratory Diseases (NCIRD). COVID-19 Omicron Variant: What You Need to Know. Accessed 27 January 2022.

12. The New York Times. Covid Updates: Israel approves a 4th dose of Covid vaccine for those with compromised immune systems. Accessed 27 January 2022.

13. World Health Organization et al. Update on Omicron. Accessed 27 January 2022.

14. Sujan Poudel, Angela Ishak, Javier Perez-Fernandez, Efrain Garcia, Darwin A León-Figueroa, Luccio Romaní, D Katterine Bonilla-Aldana, and Alfonso J Rodriguez-Morales. Highly mutated SARS-CoV-2 Omicron variant sparks significant concern among global experts-What is known so far? Travel medicine and infectious disease, 45:102234, 2022.

15. Patrick Doohan Oliver J Watson Peter Winskill Giovanni Charles Gregory Barnsley Eleanor M Riley David S Khoury Neil M Ferguson Azra C Ghani Alexandra B Hogan, Sean L Wu. Report 48: The value of vaccine booster doses to mitigate the global impact of the Omicron SARS-CoV-2 variant. Imperial College London (15-12-2021), 2021.

16. Revised Social Distancing to Apply Level 2 to Greater Seoul and Level 1 to Other Regions.

http://ncov.mohw.go.kr/en/tcmBoardView . do?brdId=12\&brdGubun=125\& dataGubun=\&ncvContSeq=366363\&contSeq=366363\&board_id=1365\&gubun= Accessed: January 27, 2021.

17. Roadmap for Gradual Recovery to a New Normal. http://ncov.mohw.go.kr/en/tcmBoardView.do?brdId=12\&brdGubun=125\& dataGubun=\&ncvContSeq=368629\&contSeq=368629\&board_id=1365\&gubun= Accessed: January 27, 2021.

18. KDCA. KDCA Press Release (Sep 7, 2021). https://www.kdca.go.kr/board/board.es?mid=a20501010000\&bid=0015\& list_no=716798\&cg_code=\&act=view\&nPage=43, 2021. Online; accessed 27 January 2021.

19. KDCA Press Release (Jan 17, 2022). https: //www.kdca.go.kr/board/board. es?mid=a20501010000\&bid=0015\& list_no=718319\&cg_code=\&act=view\&nPage=3. Accessed: January 27, 2022.

20. Hyonhee Shin. South Korea to deploy Pfizer COVID-19 pills as Omicron wave looms. Accessed 27 January 2022.

21. Zhonglei Wang and Liyan Yang. In the age of Omicron variant: Paxlovid raises new hopes of COVID-19 recovery. Journal of medical virology, 2021. 
22. Osmar Pinto Neto, Deanna M Kennedy, José Clark Reis, Yiyu Wang, Ana Carolina Brisola Brizzi, Gustavo José Zambrano, Joabe Marcos de Souza, Wellington Pedroso, Rodrigo Cunha de Mello Pedreiro, Bruno de Matos Brizzi, et al. Mathematical model of COVID-19 intervention scenarios for São Paulo-Brazil. Nature communications, 12(1):1-13, 2021.

23. Krishna P. Reddy, Kieran P. Fitzmaurice, Justine A. Scott, Guy Harling, Richard J. Lessells, Christopher Panella, Fatma M. Shebl, Kenneth A. Freedberg, and Mark J. Siedner. Clinical outcomes and cost-effectiveness of COVID-19 vaccination in South Africa. Nature Communications, 12(1), 2021.

24. Alessio Andronico, Cécile Tran Kiem, Juliette Paireau, Tiphanie Succo, Paolo Bosetti, Noémie Lefrancq, Mathieu Nacher, Félix Djossou, Alice Sanna, Claude Flamand, et al. Evaluating the impact of curfews and other measures on SARS-CoV-2 transmission in French Guiana. Nature communications, 12(1):1-8, 2021.

25. Jongmin Lee, Seok-Min Lee, and Eunok Jung. How Important Is Behavioral Change during the Early Stages of the COVID-19 Pandemic? A Mathematical Modeling Study. International Journal of Environmental Research and Public Health, 18(18):9855, 2021.

26. Chad R Wells, Jeffrey P Townsend, Abhishek Pandey, Seyed M Moghadas, Gary Krieger, Burton Singer, Robert H McDonald, Meagan C Fitzpatrick, and Alison P Galvani. Optimal COVID-19 quarantine and testing strategies. Nature communications, 12(1):1-9, 2021.

27. Michael Te Vrugt, Jens Bickmann, and Raphael Wittkowski. Effects of social distancing and isolation on epidemic spreading modeled via dynamical density functional theory. Nature communications, 11(1):1-11, 2020.

28. Quan-Hui Liu, Juanjuan Zhang, Cheng Peng, Maria Litvinova, Shudong Huang, Piero Poletti, Filippo Trentini, Giorgio Guzzetta, Valentina Marziano, Tao Zhou, Cecile Viboud, Ana I. Bento, Jiancheng Lv, Alessandro Vespignani, Stefano Merler, Hongjie Yu, and Marco Ajelli. Model-based evaluation of alternative reactive class closure strategies against COVID-19. Nature Communications, 13(1), 2022.

29. João Viana, Christiaan H van Dorp, Ana Nunes, Manuel C Gomes, Michiel van Boven, Mirjam E Kretzschmar, Marc Veldhoen, and Ganna Rozhnova. Controlling the pandemic during the SARS-CoV-2 vaccination rollout. Nature Communications, 12(1):1-15, 2021.

30. Laura Matrajt, Julia Eaton, Tiffany Leung, Dobromir Dimitrov, Joshua T Schiffer, David A Swan, and Holly Janes. Optimizing vaccine allocation for COVID-19 vaccines shows the potential role of single-dose vaccination. Nature communications, 12(1):1-18, 2021.

31. Youngsuk Ko, Jacob Lee, Yeonju Kim, Donghyok Kwon, and Eunok Jung. COVID-19 Vaccine Priority Strategy Using a Heterogenous Transmission Model Based on Maximum Likelihood Estimation in the Republic of Korea. International Journal of Environmental Research and Public Health, 18(12):6469, 2021.

32. Raphael Sonabend, Lilith K Whittles, Natsuko Imai, Pablo N Perez-Guzman, Edward S Knock, Thomas Rawson, Katy AM Gaythorpe, Bimandra A Djaafara, 
Wes Hinsley, Richard G FitzJohn, et al. Non-pharmaceutical interventions, vaccination, and the SARS-CoV-2 delta variant in England: a mathematical modelling study. The Lancet, 398(10313):1825-1835, 2021.

33. Natalie M Linton, Sung-mok Jung, and Hiroshi Nishiura. Not all fun and games: Potential incidence of SARS-CoV-2 infections during the Tokyo 2020 Olympic Games. Mathematical Biosciences and Engineering, 18(6):9685-9696, 2021.

34. Jonathan E Forde and Stanca M Ciupe. Modeling the influence of vaccine administration on COVID-19 testing strategies. Viruses, 13(12):2546, 2021.

35. Lucas Rodés-Guirao Cameron Appel Charlie Giattino Esteban Ortiz-Ospina Joe Hasell Bobbie Macdonald Diana Beltekian Hannah Ritchie, Edouard Mathieu and Max Roser. Coronavirus Pandemic (COVID-19). Our World in Data, 2020. https://ourworldindata.org/coronavirus.

36. Esteban Ortiz-Ospina Max Roser Joe Hasell Cameron Appel-Charlie Giattino Lucas Rodés-Guirao Edouard Mathieu, Hannah Ritchie. A global database of COVID-19 vaccinations. Nature Human Behaviour, 5(7):947-953, Jul 2021.

37. Rosanna C Barnard, Nicholas G Davies, Carl AB Pearson, Mark Jit, and W John Edmunds. Projected epidemiological consequences of the Omicron SARS-CoV-2 variant in England, December 2021 to April 2022. medRxiv, 2021.

38. Business Wire. Pfizer and BioNTech Provide Update on Omicron Variant. https://www.businesswire.com/news/home/20211208005542/en/, 2021. Online; accessed 27 January 2022.

39. Jennifer Couzin-Frankel. Antiviral pills could change pandemic's course. Science, 2021.

40. Elisabeth Mahase. Covid-19: Pfizer's paxlovid is $89 \%$ effective in patients at risk of serious illness, company reports. BMJ, 2021.

41. Talha Khan Burki. Omicron variant and booster COVID-19 vaccines. The Lancet Respiratory Medicine, 2021.

42. Wes Hinsley Neil Ferguson, Azra Ghani and Erik Volz on behalf of the Imperial College COVID-19 response team. Report 50 - Hospitalisation risk for Omicron cases in England. Imperial College London (22-12-2021), 2021.

43. KDCA. KDCA Press Release (January 17, 2021). https://www.kdca.go.kr/board/board.es?mid=a20501010000\&bid=0015\& list_no $=718318 \& c g$ code $=\& a c t=v i e w \& n P a g e=3,2021$. Online; accessed 27 January 2022.

44. Korean Ministry of health and welfare. Coronavirus (COVID-19), Republic of Korea. http://ncov.mohw.go.kr/en/, 2022. Online; accessed 27 January 2021.

45. KDCA. Daily vaccination situation. https://ncv.kdca.go.kr/vaccineStatus.es?mid=a11710000000, 2021. Online; accessed 27 January 2021.

46. Billy J. Gardner and A. Marm Kilpatrick. Estimates of reduced vaccine effectiveness against hospitalization, infection, transmission and symptomatic disease of a new SARS-CoV-2 variant, Omicron (B.1.1.529), using neutralizing antibody titers. medRxiv, 2021. 
47. Vivek Gupta, Rahul C Bhoyar, Abhinav Jain, Saurabh Srivastava, Rashmi Upadhayay, Mohamed Imran, Bani Jolly, Mohit Kumar Divakar, Disha Sharma, Paras Sehgal, et al. Asymptomatic reinfection in 2 healthcare workers from India with genetically distinct severe acute respiratory syndrome coronavirus 2. Clinical Infectious Diseases, 73(9):e2823-e2825, 2021.

48. Philippe Colson, Michael Finaud, Nicolas Levy, Jean-Christophe Lagier, and Didier Raoult. Evidence of SARS-CoV-2 re-infection with a different genotype. Journal of Infection, 82(4):84-123, 2021.

49. Philippe Brouqui, Philippe Colson, Cléa Melenotte, Linda Houhamdi, Marielle Bedotto, Christian Devaux, Philipe Gautret, Matthieu Million, Philippe Parola, Didier Stoupan, et al. COVID-19 re-infection. European journal of clinical investigation, 51(5), 2021.

50. Paola Cristina Resende, João Felipe Bezerra, RHTd Vasconcelos, Ighor Arantes, Luciana Appolinario, Ana Carolina Mendonça, Anna Carolina Paixao, Ana Carolina Duarte Rodrigues, Thauane Silva, Alice Sampaio Rocha, et al. Spike E484K mutation in the first SARS-CoV-2 reinfection case confirmed in Brazil, 2020. Virological [Internet], 10, 2021.

51. Md Arif Billah, Md Mamun Miah, and Md Nuruzzaman Khan. Reproductive number of coronavirus: A systematic review and meta-analysis based on global level evidence. PloS one, 15(11):e0242128, 2020.

52. Finlay Campbell, Brett Archer, Henry Laurenson-Schafer, Yuka Jinnai, Franck Konings, Neale Batra, Boris Pavlin, Katelijn Vandemaele, Maria D Van Kerkhove, Thibaut Jombart, et al. Increased transmissibility and global spread of SARS-CoV-2 variants of concern as at June 2021. Eurosurveillance, 26(24):2100509, 2021.

53. Yaping Wang, Ruchong Chen, Fengyu Hu, Yun Lan, Zhaowei Yang, Chen Zhan, Jingrong Shi, Xizi Deng, Mei Jiang, Shuxin Zhong, et al. Transmission, viral kinetics and clinical characteristics of the emergent SARS-CoV-2 Delta VOC in Guangzhou, China. EClinicalMedicine, 40:101129, 2021.

54. World Health Organization et al. Transmission of SARS-CoV-2: implications for infection prevention precautions: scientific brief, 09 July 2020. Technical report, World Health Organization, 2020.

55. Moran Ki et al. Epidemiologic characteristics of early cases with 2019 novel coronavirus (2019-nCoV) disease in Korea. Epidemiology and health, 42, 2020.

56. The effectiveness for severity and death of COVID-19 vaccine from May to July 2021. https://www.kdca.go.kr/board/board.es?mid=a20602010000\&bid= 0034\&list_no=716913\&act=view. Accessed: January 27, 2022.

57. Yong-Hoon Lee, Chae Moon Hong, Dae Hyun Kim, Taek Hoo Lee, and Jaetae Lee. Clinical course of asymptomatic and mildly symptomatic patients with coronavirus disease admitted to community treatment centers, South Korea. Emerging infectious diseases, 26(10):2346, 2020.

58. The weekly news review (January 21, 2022). https://www. seoul.go.kr/coronaV/coronaStatus.do?menu_code=07. Accessed: January 27, 2022. 
59. Differential payment of loss compensation to increase the turnover rate of severe patient beds. http://ncov.mohw.go.kr/tcmBoardView.do?brdId=\&brdGubun= \&dataGubun=\&ncvContSeq=368962\&contSeq=368962\&board_id=\&gubun=ALL. Accessed: January 27, 2022.

60. Notice regarding the release of quarantine for COVID-19 and the order to move the hospital bed.

http://ncov $\cdot$ mohw $\cdot$ go $\cdot \mathrm{kr} / \mathrm{tcmBoardView} \cdot$ do?brdId=\&brdGubun=\&dataGubun= \&ncvContSeq=369074\&contSeq=369074\&board_id=\&gubun=ALL. Accessed: January 27, 2022.

61. UKHSA (UK Health Security Agency). COVID-19 vaccine weekly surveillance report week 1. https://assets.publishing.service.gov.uk/government/ uploads/system/uploads/attachment_data/file/1045329/Vaccine_ surveillance_report_week_1_2022.pdf, January 2022.

62. KDCA Press Release (May 18, 2021). https://www.kdca.go.kr/board/board.es?mid=a20501010000\&bid=0015\& list_no=713349\&cg_code=\&act=view\&nPage=1. Accessed: January 27, 2022.

63. KDCA Press Release (Dec 1, 2021). https://www.kdca.go.kr/board/board.es?mid=a20501010000\&bid=0015\& list_no=717719\&cg_code=\&act=view\&nPage=1. Accessed: January 27, 2022.

64. Arin Kim. South Korea finds first cases of omicron. http://www.koreaherald.com/view.php?ud=20211201000944, 2021. Online; accessed 20 December 2021.

65. Odo Diekmann, JAP Heesterbeek, and Michael G Roberts. The construction of next-generation matrices for compartmental epidemic models. Journal of the Royal Society Interface, 7(47):873-885, 2010.

66. Maia Martcheva. An introduction to mathematical epidemiology, volume 61. Springer, 2015.

67. Elisabeth Mahase. Covid-19: Hospital admission 50-70\% less likely with omicron than delta, but transmission a major concern. BMJ, 2021.

68. Henning Gruell, Kanika Vanshylla, Pinkus Tober-Lau, David Hillus, Philipp Schommers, Clara Lehmann, Florian Kurth, Leif Erik Sander, and Florian Klein. mRNA booster immunization elicits potent neutralizing serum activity against the SARS-CoV-2 Omicron variant. medRxiv, 2021.

69. Wilfredo F Garcia-Beltran, Kerri J St Denis, Angelique Hoelzemer, Evan C Lam, Adam D Nitido, Maegan L Sheehan, Cristhian Berrios, Onosereme Ofoman, Christina C Chang, Blake M Hauser, et al. mRNA-based COVID-19 vaccine boosters induce neutralizing immunity against SARS-CoV-2 Omicron variant. medRxiv, 2021.

\section{Acknowledgements}

This paper is supported by the Korea National Research Foundation (NRF) grant funded by the Korean government (MEST) (NRF-2021M3E5E308120711). This paper is also supported by the Korea National Research Foundation (NRF) grant funded by the Korean government (MEST) (NRF-2021R1A2C100448711). 


\section{Competing Interests}

The authors declare no competing interests.

\section{Author contributions}

Jongmin Lee - conceptualization, draft preparation, methodology, software. Renier Mendoza, Victoria May P. Mendoza, and Youngsuk Ko - conceptualization, draft preparation, methodology. Jacob Lee and Yubin Seo - conceptualization, draft preparation. Eunok Jung, conceptualization, draft preparation, methodology, project supervision. All authors read and approved the final version of the manuscript. 


\section{Supplementary Files}

This is a list of supplementary files associated with this preprint. Click to download.

- Supplementaryfile.pdf 